easily be adjusted, improving the true positive detection rate at the expense of more false positive indications.

Of course, we must not get too carried away by this apparent ability to automatically classify by painless learning processes, for while the computing specialist has always seen the necessity to express decision rules in terms of mathematics or logic, the statistician also has an armoury of classification tools able to produce results often as convincing as those of the ANN. Indeed, there is much to commend using the techniques of multivariate distributions and principal component analysis as a silver standard, after the gold standard of the expert, by which to evaluate the new methods. Analytically, the ANN only drives a decision surface through the data, and statisticians claim to have been doing just that for a very long time.

Although it will also be a long time before artificial neural networks approach the complexity or performance of their human counterparts, and in this respect the quoted receiver operator coefficient (ROC) curves often show there is room for improvement, it is clear that the processes of decision making we use on a day to day basis embarrassingly defy an exclusively algorithmic synthesis. The fact that biological systems achieve such impressive feats of information processing using a basic, simplistic, connec- tionist approach will remain a constant source of inspiration. While it would be unwise to speculate on technical developments in this field, there can be little doubt that the future will be an exciting one.

P UNDRILI

Department of Bio-Medical Physics and Bio-Engineering, University of Aberdeen Medical School,

Foresterhill, Aberdeen AB25 2ZD

1 McCulloch WS, Pitts W. A logical calculus of the ideas immanent in nervous activity. Bull Math Biophys 1943;5:115-33.

2 Hopfield JJ. Neural networks and physical systems with emergent collective computational abilities. Proc Natl Acad Sci USA 1984;79:2554-8.

3 Mutlukan E, Keating D. Visual field interpretation with a personal computer based neural network. Eye 1994;8:321-3.

4 Spenceley SE, Henson DB, Bull DR. Visual field analysis using artificial neural networks. Ophthal Physiol Opt 1994;14:239-48.

5 Brigatti L, Hoffman D, Caprioli J. Neural networks to identify glaucoma with structural and functional measurements. Am f Ophthalmol 1996;121: 511-21.

6 Goldbaum MH, Sample PA, White H, Colt B, Raphaelian P, Fechtner RD, et al. Interpretation of automated perimetry for glaucoma by neura network. Invest Ophthalmol Vis Sci 1994;35:3362-73.

7 Madsen EM, Yolton RL. Demonstration of a neural network expert system for recognition of glaucomatous visual field changes. [Review] Mil Med 1994;159:553-7.

8 Frame A, Cree MJ, Olson J, Undrill PE. Computer based classification of retinal microaneurysms. Proceedings of International Conference on Neural Networks and Expert Systems in Health Care, Plymouth, 1996.

\title{
Lymphocyte subsets and ocular inflammation: future prospects for immune deviation therapy?
}

In the early 1960 s the cells of the immune system were simply divided into the $\mathrm{B}$ cells and the $\mathrm{T}$ cells. $\mathrm{B}$ cells were responsible for antibody production whereas $T$ cells produced a wide variety of soluble factors designated as lymphokines. Subdivision of T cells became possible with the development of the technique to produce monoclonal antibodies against a wide variety of proteins including cell surface components. This led to a classification of $\mathrm{T}$ cells based on the presence of the so called markers CD4 or CD8. Analysis of these two types of $T$ cells resulted in a functional separation of $\mathrm{T}$ cell populations into $\mathrm{CD} 4$ positive $\mathrm{T}$ cells providing a helper function for the differentiation of antibody producing B cells and CD8 positive T cells which were shown to kill target cells and thus were named cytotoxic $T$ cells. Recently it has become clear, however, that such a strict functional division is not valid any more and that certain properties of CD4 and CD8 T cells show an overlap. CD4 cells interact with antigen presenting cells via MHC class II (HLA-DR) molecules, whereas CD8 cells recognise antigen in the context of MHC class I (HLA-A, B, or C).

It soon appeared that the membrane markers were not sufficient to characterise the various functions of $T$ cells, and a further breakthrough came from the functional and molecular characterisation of the many lymphokines produced by these cells. In the late 1980s Mosman and Coffman ${ }^{2}$ presented a division of the $\mathrm{T}$ helper cells into Th1 and Th2 populations, depending upon their lymphokine (cytokine) production profile. The balance between a Th1 or a Th2 response is of great importance in the outcome of the immune defence mechanisms employed to combat infectious disease, and may also influence the occurrence of allergic (conjunctivitis) or autoimmune disease. ${ }^{3}$ This has been nicely illustrated in a mouse model of leishmaniasis whereby mouse strains that react to this infection with a Th2 type response succumb, whereas animals showing a Th1 reactivity survive.
Deviation of the Th2 response in these mouse strains to a Th1 response leads to protection against the Leishmania parasite.

A large body of evidence now shows that a Th1 response is primarily responsible for a delayed type hypersensitivity response and is characterised by a high production of interferon $\gamma$ and IL-2 and a low or absent release of IL-4 (see Table 1). The Th2 cells, on the other hand, produce large amounts of IL-4 and IL-5 and play a key role in humoral immunity including the IgE response. The cytokines produced by the Th2 cells have a deactivating effect on macrophages and thus counteract the tissue destruction which is characteristic of the Th1 response. An exaggerated Th2 response is associated with allergic disease and immunoglobulin mediated autoimmunity, whereas a Th1 response is held responsible for contact hypersensitivity and certain autoimmune diseases such as rheumatoid arthritis. It has been speculated that several organ specific autoimmune diseases are caused by interferon $\gamma$ producing Th1 cells. These findings have led to new approaches for the immunotherapy of inflammatory autoimmune disease which are based on a deviation of the harmful Th1 response towards an immunosuppressive Th2 response.

Our knowledge concerning the factors that regulate the balance between $\mathrm{Th} 1 / \mathrm{Th} 2$ is rapidly expanding. Th2 cell proliferation is inhibited by interferon $\gamma$ and Th1 cell proliferation is suppressed by IL-4. Other cytokines that play a regulatory role are IL-10 and IL-12 which are both produced by antigen presenting cells. IL-10 supports the Th2 response and IL-12 the Th1 response. The microenvironment may dictate whether an antigen presenting cell produces IL-10 or IL-12 thus leading to either a Th1 or a Th2 response. Administration of antigens via the mucosal immune system is thought to induce a Th2 response and so called oral tolerisation strategies are currently being explored to treat a number of putative autoimmune 
Table 1 Properties of the Th1 and Th2 immune response

\begin{tabular}{lll}
\hline & Th1 & Th2 \\
\hline $\begin{array}{c}\text { Characteristic } \\
\text { cytokine }\end{array}$ & Interferon $\gamma$ & IL-4 \\
& IL-12 & IL-5 \\
& IL-2 $\alpha / \beta$ & IL-6 \\
& TNF $\alpha / 10$ \\
Action & Delayed type hypersensitivity & II-13 \\
& Immediate type \\
& Complement binding antibodies & $\begin{array}{l}\text { Non-complitement binding } \\
\text { antibodies }\end{array}$ \\
Beneficial & Intracellular organisms (leprosy, & $\begin{array}{l}\text { Suppresion autoimmunity } \\
\text { (arthritis, uveitis?) }\end{array}$ \\
Harmful & Leishmania, Toxoplasma) & Allergic disease (asthma, \\
effect & Autoimmune disease & allergic conjunctivitis) \\
& Intraocular inflammation & Intracellular organisms \\
\hline
\end{tabular}

diseases such as multiple sclerosis, rheumatoid arthritis, and uveitis. ${ }^{4}$ Other options include a manipulation of the cytokines involved in the differentiation of $\mathrm{T}$ lymphocytes.

For the eye, experimental evidence also clearly points to a major role of a Th1 type response in the pathogenesis of autoimmune uveitis. ${ }^{5}$ Supporting evidence for such a mechanism in the pathogenesis of clinical uveitis is not very strong yet. But, in this month's issue of the $B \mathcal{B O}$ (p 1002), Japanese investigators provide evidence for a Th1 type autoimmune response in Vogt-KoyanagiHarada (VKH) disease.

VKH is a bilateral acute panuveitis common in Japan that is considered to be caused by an autoimmune reaction directed against melanocytes. In 1982 Maezawa et $a l^{6}$ showed that $\mathrm{VKH}$ patients had circulating cytotoxic $\mathrm{T}$ cells that were able to kill a P-36 melanoma cell line, which contains antigens that cross react with normal melanocytes, and indicated that the reaction was HLA-DR restricted, which may explain the strong association of this disease with HLA-DR4. Norose and Yano have extended these observations and have now established melanoma specific CD4 positive cytotoxic $T$ cell lines from the peripheral blood of two patients with $\mathrm{VKH}$, while failing to develop such cells when culturing leucocytes obtained from healthy donors. By analysing the cytokine production profile they showed that these cells were high producers of interferon $\gamma$ and IL-2, but did not release IL-4, which allowed them to draw the conclusion that these cells belonged to the Th1 type of $\mathrm{T}$ lymphocytes. Of interest is their observation that the generated cytotoxic $\mathrm{T}$ cell lines all carried the CD4 marker, which was originally not considered to be associated with target killing properties. Since CD4 cells can only recognise antigen when it is presented to them via HLA class II molecules this may explain the strong association observed between HLA-DR4 and VKH.

Ultimate proof of the role of the Th1 type autoimmune reactivity against melanocytes in $\mathrm{VKH}$ and, possibly other uveitis entities, will come from the analysis of the specificity and phenotype of $T$ cells obtained from the inflamed ocular tissue or its surrounding media, an approach that is currently being undertaken in ocular toxoplasmosis. ${ }^{7}$ If this also supports a major role for interferon $\gamma$ producing Th1 cells, further therapeutic strategies may be developed to deviate this response to a more benign interleukin 4 producing Th2 phenotype.

AIZE KIJLSTRA

Netherlands Ophthalmic Research Institute, and Department of Ophthalmology, University of Amsterdam, Amsterdam, the Netherlands

1 Mizuki N, Inoko H, Ohno S. Role of HLA and T lymphocytes in the immune response. Ocular Immunology and Inflammation 1994;2:57-91.

2 Mosman TR, Coffman RL. Th1 and Th2 cells: different patterns of lymphokine secretion lead to different functional properties. Annu Rev Immunol 1989;7:145-73.

3 Paul WE, Seder RA. Lymphocyte responses and cytokines. Cell 1994;76: 241-51.

4 Weiner HL, Friedman A, Miller A, Khoury SJ, al-Sabbagh A, Santos E, et al. Oral tolerance; immunologic mechanisms and treatment of animal and human organ-specific auto immune diseases by oral administration of antihuman organ-specific auto immune diseases

gens. Annu Rev Immunol 1994;12:809-37.
5 Gery I, Streilein JW. Autoimmunity in the eye and its regulation. Curr Opin Immunol 1994;6:938-45.

6 Maezawa N, Yano A, Taniguchi M, Kojima S. The role of cytotoxic T lymphocytes in the pathogenesis of Vogt-Koyanagi-Harada disease. Ophthalmologica 1982;185:179-86.

7 Feron EJ, Verjans GM, Klaren VN, van Doornik CE, Luyendijk L, Kijlstra A. Antigen specificity of intraocular T cells in recurrent ocular toxoplasmosis. Invest Ophthalmol Vis Sci 1995;36:S611. 